Médecins de garde: les médecins de premier recours pour les soins urgents

Le Forum du sauvetage de la FMH réunit les sociétés de discipline médicale participant à l'assistance préhospitalière d'urgence; c'est pourquoi I'Interassociation de sauvetage et le Service sanitaire coordonné de l'armée y sont également représentés. Les lignes directrices de la FMH sur le sauvetage en Suisse, publiées en août 2010, visent à uniformiser les soins professionnels des premiers secours sur les plans opérationnel et juridique et couvrent également les catastrophes et autres événements graves. La FMH s'engage pour que les soins urgents soient assurés par les urgentistes, ou des généralistes spécialement formés, et les ambulanciers. L'expérience montre qu'il est de plus en plus difficile de motiver les nouvelles générations de médecins pour le service d'urgence; et pourtant, la participation à ces services est un des devoirs, mais aussi l'activité centrale, des médecins de premier recours. Comme il n'est pas possible aujourd'hui de penser les soins urgents sans $y$ associer les médecins de famille implantés, les responsables de l'Institut suisse pour la formation médicale postgraduée et continue (ISFM) ont intégré le cours pour médecin de garde dans l'offre de formation destinée aux médecins de premier recours, tandis que le Forum du sauvetage de la FMH soutient ces derniers dans leur fonction de médecin de garde. Pour endiguer la pénurie de médecins de premier recours qui se profile en médecine d'urgence, le monde politique doit de toute urgence revaloriser la médecine de famille dans sa prise en charge des patients devant bénéficier de soins urgents.

Dr Ernst Gähler, vice-président, responsable du Forum du sauvetage de la FMH

\title{
Médecins de garde: médecins de premier recours pour les soins urgents et partenaires indispensables du sauvetage
}

Bruno Durrera,

Wolfgang Ummenhofer ${ }^{b}$,

Heinz Zimmermann

(ordre alphabétique)

a Dr, médecine générale $\mathrm{FMH}$ urgentiste SSMUS, médecine du sport SSMM,

Lauterbrunnen

b Prof., service d'anesthésie, hôpital universitaire de Bâle

c Prof., directeur du service des urgences, hôpital de l'Île, Berne
Correspondance:

Prof. Dr Wolfgang Ummenhofer Hôpital universitaire de Bâle Service d'anesthésie

CH-4031 Bâle

wummenhofer@uhbs.ch
Légalement, les cantons sont dans l'obligation d'assurer en tout lieu et en tout temps la prise en charge des soins qui relèvent de la médecine d'urgence. Dans le domaine des soins de premier recours, ils ont délégué cette tâche aux sociétés cantonales de médecine.

Dans les régions rurales ou de montagne qui ne bénéficient d'aucun réseau de médecine d'urgence, les médecins de premier recours ayant accompli une formation de médecin de garde sont tenus d'intervenir en situation d'urgence avant l'arrivée des secours terrestres ou aériens lorsque le diagnostic vital d'un patient est engagé. Sous la supervision de la $\mathrm{FMH}$, la Société suisse de médecine d'urgence et de sauvetage (SSMUS) propose un cours de médecin de garde (www.fmh.ch $\rightarrow$ SERVICES $\rightarrow$ Forum du sauvetage $\rightarrow$ Médecin de garde - service d'urgence médicale). La formation des urgentistes se distingue principalement de celle des médecins de garde par l'invasivité des mesures précliniques enseignées. Toutefois, les agglomérations profitent également d'un lien entre ces deux spécialisations car même une visite à domicile anodine peut se transformer en situation d'urgence. Par ailleurs, en vue d'améliorer le tri des patients en situation d'urgence, il faut approfondir la collaboration entre les acteurs qui réceptionnent les appels sanitaires.

En dehors des heures d'ouverture des cabinets, les centrales d'appels des médecins peuvent assurer le tri des urgences qui peuvent être traitées en ambulatoire et pour lesquelles aucun diagnostic vital n'est engagé, et les adresser à la permanence de médecine de pre- mier recours la plus proche. A cet effet, il existe des services d'urgence autonomes assurés par des médecins de famille qui collaborent en partie avec les services d'urgence des hôpitaux.

Lorsque les jours du patient sont potentiellement menacés, l'alarme doit être donnée au 144. La centrale peut ensuite parfaitement coordonner l'intervention des premiers membres de la chaîne du sauvetage (non professionnels et secouristes) avec celle des médecins de garde et du sauvetage aérien ou terrestre.

Mais la prise en charge de la population dans les situations d'urgence - une compétence clé de la médecine de famille - est de plus en plus compromise

Dans son rôle de forum des sociétés de discipline, le Forum du sauvetage de la FMH a élaboré des lignes directrices pour une assistance préhospitalière d'urgence uniforme et obligatoire, également en cas d'événements de grande ampleur. La FMH s'engage pour que les soins urgents soient assurés par les urgentistes, ou des généralistes spécialement formés, et les ambulanciers. En été 2010, le Forum du sauvetage a actualisé ses thèses publiées en 1996 (Bulletin des médecins suisses $n^{\circ} 33,2010$ et www.fmh.ch $\rightarrow$ SERVICES $\rightarrow$ Forum du sauvetage). Le BMS consacrera une série $d^{\prime}$ articles périodiques qui traiteront plus en détail les huit points énoncés dans les nouvelles lignes directrices. 
par la pénurie de médecins de famille qui se profile. La baisse drastique du nombre de médecins de famille complique de plus en plus les services d'urgence dans les régions excentrées et réduit l'attrait de la profession, notamment pour les femmes avec famille. Les jeunes médecins ne sont plus disposés à maintenir gratuitement un service disponible $24 \mathrm{~h} / 24$, lorsqu'en même temps on assiste à une réduction de la rémunération des prestations. Pourtant, le concept de médecin de garde ne peut fonctionner qu'en leur présence. Nous risquons de devoir faire face à une pénurie subaiguë de soins relevant de la médecine d'urgence dans les régions périphériques, qui, à moyen et à long terme, ne pourra plus être enrayée par des médecins venus de l'étranger.

Le monde politique doit contrer cette tendance: tout d'abord, il faut une déclaration d'intention claire de la part des décideurs politiques pour renforcer les médecins de premier recours et continuer de les impliquer dans la médecine d'urgence, notamment dans les régions périphériques. En outre, les cantons doivent indemniser le cours de médecin de garde, le piquet et l'équipement d'urgence nécessaire aux médecins de premier recours qui assument un service de garde. A l'avenir, les soins de premier recours et les soins urgents dans les régions de campagne ou de montagne seront assurés par les cabinets de groupe, les centres médicaux et de santé organisés dans de plus grandes agglomérations. Les cabinets délaissés des zones périphériques pourront servir de dispensaires gérés par les infirmières municipales et les services d'aide et de soins à domicile ou par les assistantes médicales. Ces dispensaires seront en liaison étroite avec le centre de médecins de premier recours le plus proche, qui en assume la responsabilité.

Le Forum du sauvetage soutient les médecins de premier recours dans leur fonction de médecin de garde. Pour endiguer la pénurie qui se profile en médecine d'urgence, le monde politique doit de toute urgence revaloriser la médecine de famille dans sa prise en charge des patients devant bénéficier de soins urgents. A cet effet, l'enseignement dispensé à l'université doit mettre encore plus l'accent sur les connaissances et les aptitudes requises en médecine d'urgence. La FMH s'attelle à ce que les contenus du cours de médecin de garde soient incorporés au cursus des facultés de médecine.

\section{En bref}

1. Il faut une déclaration d'intention claire de la part des décideurs politiques pour renforcer les compétences des médecins de premier recours en matière de médecine d'urgence et continuer de les impliquer dans cette dernière.

2. Que ce soit dans les cabinets médicaux implantés en ville ou à la campagne, les médecins de premier recours peuvent intervenir en attendant l'arrivée des secours aériens ou terrestres.

3. Dans les régions périphériques, les cantons peuvent faire appel aux médecins de premier recours ayant accompli la formation de médecin de garde lorsque le diagnostic vital d'un patient est engagé. Le Forum du sauvetage de la FMH soutient un tel système de médecins de garde avec une rémunération adaptée, et demande que le monde politique reconnaisse et revalorise la médecine de famille.

4. Dans ce but, les futurs médecins doivent acquérir les compétences de base en médecine d'urgence déjà au cours de leurs études de médecine. La FMH s'attelle à ce que les contenus du cours de médecin de garde soient incorporés aux cursus des facultés de médecine. 